\title{
Abordagem fisioterapêutica da incontinência urinária em idosos na atenção primária em saúde
}

\author{
Physiotherapeutic approach for urinary incontinence in older adults in primary health care \\ Enfoque fisioterapéutico de incontinencia urinaria en ancianos en la atención primaria de salud \\ Crislainy Vieira Freitas', Ilva Lana Balieiro Capela², Sandy Amara Costa Silva de Caldas ${ }^{3}$, \\ Thais Monteiro Góes Almeida ${ }^{4}$
}

\begin{abstract}
RESUMO I O objetivo deste estudo foi avaliar os benefícios da abordagem fisioterapêutica da IU em idosos em uma unidade básica de saúde (UBS) de Belém (PA). Trata-se de uma proposta para treinamento dos músculos do assoalho pélvico (MAP) em 10 encontros semanais com atividades progressivas. Foram incluídos idosos de ambos os sexos, e excluídos os indivíduos com prejuízo cognitivo, instabilidade hemodinâmica e mobilidade impossibilitada, além dos idosos com participação inferior a 50\% dos encontros. Foi realizada avaliação por meio de dados sociodemográficos e clínicos, e da QV, por meio do King's Health Questionaire (KHQ), que foi reaplicado ao final dos encontros. A amostra inicial contou com 10 idosos, com idade de 70,3 $\pm 5,01$ anos, sendo $80 \%$ do sexo feminino, $80 \%$ dos idosos relataram cirurgias pélvicas prévias, prolapsos em 3 idosos, destes, 2 eram de bexiga. Os domínios do $\mathrm{KHQ}$ com escores mais altos, e

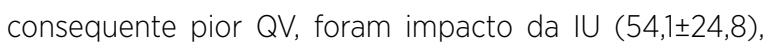
percepção de saúde $(43,7 \pm 11,5)$ e medidas de gravidade $(31,2 \pm 23,8)$. Houve significância estatística em vários domínios da QV dos idosos submetidos a abordagem fisioterapêutica, demonstrando que, mesmo em um nível mais baixo de atenção à saúde é possível ter efeitos relevantes sobre a IU e a QV desse público.

Descritores I Idoso; Incontinência Urinária; Fisioterapia; Atenção Primária à Saúde; Qualidade de Vida.
\end{abstract}

ABSTRACT I The aim of this study was to evaluate the benefits of the physiotherapeutic approach to urinary incontinence (UI) in the older population in a health center of Belém (PA). This is a proposal for strengthening pelvic floor muscles (PFM) in ten weekly meetings with progressive activities. Patients of both genders were included, and individuals with cognitive impairment, hemodynamic instability and immobility were excluded, as well as those with less than 50\% participation in the meetings. Sociodemographic and clinical data were evaluated and quality of life (QoL) was analyzed through the King's Health Questionnaire (KHQ), which was reapplied at the end of the meetings. The initial sample consisted of ten older adults, aged $70.3 \pm 5.01$ years, $80 \%$ female; $80 \%$ of the sample reported previous pelvic surgeries, three reported prolapses, of which two were in the bladder. The KHQ domains with higher scores and consequent worse QoL were the impact of UI (54.1 \pm 24.8$)$, health perception ( $43.7 \pm 11.5)$, and severity measures (31.2 \pm 23.8$)$. There was statistical significance in several domains of QoL of the patients undergoing the physiotherapeutic approach, demonstrating that even in a lower level of health care it is possible to have relevant effects on the UI and QoL of this public.

Keywords I Older population; Urinary incontinence; Physical Therapy; Primary Health Care; Quality of life.

RESUMEN I El objetivo de este estudio fue evaluar los beneficios del enfoque fisioterapéutico de la incontinencia urinaria (IU) en ancianos de una Unidad Básica de Salud (UBS) en Belém (PA, Brasil). Este enfoque consiste en una propuesta de entrenamiento de los músculos del suelo pélvico (MSP)

Estudo desenvolvido na Unidade Municipal de Saúde, Secretaria Municipal de Saúde, Belém, Pará, Brasil.

'Instituto Federal de Educação, Ciência e Tecnologia do Pará (IFPA) - Belém (PA), Brasil. E-mail: crislainyvieira@gmail.com. Orcid: 0000-0001-5477-2426

¿Universidade Federal do Pará (UFPA) - Belém (PA), Brasil. E-mail: lanacapela@hotmail.com. Orcid: 0000-0003-1891-708X

3Universidade Federal do Pará (UFPA) - Belém (PA), Brasil. E-mail: sandyamaracosta@gmail.com. Orcid: 0000-0002-1616-1584

${ }^{4}$ Instituto Federal de Educação, Ciência e Tecnologia do Pará (IFPA) - Belém (PA), Brasil. E-mail: thais.goes@ifpa.edu.br.

Orcid: 0000-0003-0886-2564 
durante 10 sesiones semanales con actividades progresivas. Se incluyeron a ancianos de ambos sexos, y se excluyeron a personas con deterioro cognitivo, inestabilidad hemodinámica y movilidad reducida, además de los ancianos con menos del $50 \%$ en asistencia a las reuniones. La evaluación se llevó a cabo con datos sociodemográficos y clínicos y de calidad de vida (CV) utilizando King's Health Questionnaire (KHQ), que lo volvieron a aplicar al final de las reuniones. La muestra inicial estuvo formada por 10 ancianos de 70,3ะ5,01 años; el 80\% de ellos eran del sexo femenino y el $80 \%$ informaron haber realizado cirugías pélvicas previas, con prolapsos en 3 ancianos; de estos, 2 eran de vejiga. Los dominios KHQ con las puntuaciones más altas y, en consecuencia, la peor calidad de vida fueron el impacto de la IU $(54,1 \pm 24,8)$, de la percepción de la salud $(43,7 \pm 11,5)$ y de medidas de gravedad $(31,2 \pm 23,8)$. Hubo una significación estadística en varios dominios de CV de los ancianos que se sometieron al enfoque fisioterapéutico, lo que demuestra que incluso en un nivel más bajo de asistencia sanitaria es posible generar efectos relevantes sobre la IU y la CV de esta población. Palabras clave I Anciano; Incontinencia Urinaria; Fisioterapia; Atención Primaria de Salud; Calidad de Vida.

\section{INTRODUÇÃO}

O aumento da expectativa de vida da população idosa é uma realidade atual no Brasil, contudo, junto a esse processo, nota-se a maior ocorrência de doenças crônicas e as chamadas síndromes geriátricas, como a incontinência urinária (IU), condição associada a causas multifatoriais, que pode repercutir na independência, mobilidade e qualidade de vida dos idosos ${ }^{1-3}$.

Definida como a incapacidade de armazenar e reter urina com consequente perda acidental e involuntária, a IU está quase sempre erroneamente interpretada como parte natural do envelhecimento devido a sua alta ocorrência nessa população, estando frequentemente relacionada à sub-relato por parte dos próprios idosos e, muitas vezes, da equipe de saúde ${ }^{1,4}$.

Em 2014, a Associação Portuguesa de Urologia ${ }^{5}$ apontou que $33 \%$ das mulheres e $16 \%$ dos homens, com mais de 40 anos têm sintomas da doença. Os tipos de IU mais comuns são incontinência urinária de urgência (IUU) que é antecipada por um desejo repentino de urinar difícil de inibir; incontinência urinária de esforço (IUE) ocasionada por espirro, tosse, ou outras situações de esforço físico; e incontinência urinária mista (IUM), a combinação das duas formas anteriores. Há também a incontinência funcional (IUF), secundária a fatores que não são do trato urinário, como problemas musculoesqueléticos, psicológicos e fatores ambientais ${ }^{2}$.

Embora não seja condição potencialmente letal, a IU oferece grandes riscos à qualidade de vida do idoso, levando ao isolamento social, ansiedade e depressão, aumento de risco de quedas e fraturas (IUU), além de hospitalizações e admissões em instituição de longa permanência ${ }^{2}$. Torna-se imprescindível a abordagem preventiva e tratamento precoce para IU, o que é possível desde a atenção primária a saúde (APS) ${ }^{6}$ e é nesse nível que se podem desenvolver ações em relação aos sintomas da IU, com intuito de minimizar as complicações e danos à saúde e à funcionalidade ${ }^{7}$.

As intervenções conservadoras são as opções terapêuticas mais recomendadas, pois envolvem menor custo financeiro e baixo risco de efeitos colaterais, dentre as quais, o treinamento dos músculos do assoalho pélvico (MAP), conduzido por fisioterapeuta, é considerado de primeira linha ${ }^{8}$. Apesar disso, no Brasil, esse tratamento não é usualmente realizado APS e faltam dados na literatura científica sobre o tema ${ }^{9}$. Torna-se necessário, deste modo, oferecer medidas educativas e o treino muscular a idosos ainda no contexto da APS. Assim, este estudo teve por objetivo avaliar os benefícios da abordagem fisioterapêutica da IU em idosos em uma unidade básica de saúde (UBS) de Belém (PA).

\section{METHODOLOGY}

Trata-se de um estudo longitudinal prospectivo com intervenção e abordagem quantitativa, realizado em uma UBS do município de Belém, que teve como proposta a abordagem da IU por meio do fortalecimento dos MAP ou períneo propostos em 10 encontros semanais com duração de uma hora cada. Foram incluídos no estudo idosos de ambos os sexos que utilizavam os serviços da UBS, sendo excluídos os idosos com prejuízo cognitivo, instabilidade hemodinâmica ou com mobilidade impossibilitada. Os participantes com frequência inferior a $50 \%$ nos encontros propostos também foram excluídos dos resultados.

Primeiramente foi realizada uma ação educativa sobre o tema, na qual participaram 25 idosos, destes, 
10 manifestaram disponibilidade para participar do grupo. No primeiro encontro foi realizada uma abordagem educativa e avaliação individual, por meio de entrevista estruturada contendo dados sociodemográficos, clínicos e outros fatores de risco ou proteção para IU, e o King's Health Questionaire (KHQ), um instrumento elaborado para avaliar a qualidade de vida (QV) de idosos com IU validado para mulheres brasileiras por Fonseca et al. ${ }^{10}$.

Do segundo ao oitavo encontros foram propostos exercícios estáticos para treino dos MAP, abrangendo treino proprioceptivo e respiratório, inicialmente na posição de decúbito dorsal, passando para sedestação e bipedestação, a fim de aproxima-los das posturas do cotidiano. Os idosos receberam uma cartilha tais exercícios, a fim de reforçar o aprendizado dos exercícios.

No nono encontro foram propostos exercícios dinâmicos que simulassem atividades diárias associadas ao treino dos MAP (caminhar associado à contração dos MAP com e sem carga, subir e descer escadas, sentar e levantar de cadeira) e o treino da contração em situação de tosse simulada. O último encontro foi destinado à reavaliação dos participantes com os mesmos instrumentos utilizados inicialmente.

Após o término dos encontros, os dados foram tabulados e analisados estatisticamente por meio do software Bioestat versão 5.3. Inicialmente, comparados pelo teste de normalidade Shapiro-Wilk e, aos dados classificados em paramétricos aplicou-se a teste TStudent pareado, nos categóricos aplicou-se o teste $\mathrm{G}$, adotando p-valor $\leq$ 0,05 como nível de significância. Os dados categóricos foram representados através de frequência relativa (\%), os dados paramétricos em média, desvio padrão, mediana, máxima e mínima.

É importante declarar que este estudo seguiu aos princípios éticos de acordo com a declaração de Helsink e resoluções no 196/96 e no 510/2016 do Conselho Nacional de Saúde, contou com a prévia aceitação formal dos participantes por meio de assinatura de termo de consentimento livre e esclarecido (TCLE), além da aprovação do comitê de ética em pesquisa em seres humanos sob parecer $n^{\circ} 3.165 .816 / 2019$.

\section{RESULTADOS E DISCUSSÃO}

A amostra inicial contou com 10 idosos, com idade de 70,3 $\pm 5,01$ anos, sendo $80 \%$ do sexo feminino, $40 \%$ casados, e todos aposentados. Apenas 1 idoso com diabetes mellitus (DM) e 6 com hipertensão arterial sistêmica (HAS). Uso de medicamentos diuréticos e etilismo social em 30\% dos idosos e consumo excessivo de café em $40 \%$, ingesta hídrica de 2 litros/dia em 50\% dos participantes. Todos os idosos praticavam exercícios físicos.

Cirurgias pélvicas prévias foram apontadas por $80 \%$ dos idosos, destas houve um idoso com histórico de prostatectomia e, dentre as mulheres houve história de laqueadura, histerectomia, miomectomia e perineoplastia. Apenas 2 eram nulíparas, sendo as demais multíparas; tempo médio de menopausa de 22,12 $\pm 10,09$ anos. Prolapsos em 3 idosos, destes, 2 eram de bexiga.

Pincelia e Moccellinb ${ }^{11}$ reuniram os principais fatores que podem predispor a IU: diabetes, obesidade, tabagismo, menopausa, doenças do sistema nervoso central, uso de medicamentos, restrição da mobilidade, múltiplos partos vaginais, impactação fecal, distúrbios psíquicos, diminuição do tônus da região perineal e uso de álcool, alguns dos quais presentes neste presente estudo. No caso dos homens, Abrams et al. ${ }^{12}$ associam a prostatectomia a ocorrência de IU, geralmente a IUF, semelhante ao que ocorreu em um dos sujeitos deste estudo.

Dos 10 idosos, 9 apresentaram queixa urinária, destas houve relato característico de IUU (4), IUE (3), IUM (1) e IUF (1). Dois idosos foram excluídos dos resultados, um devido à ausência de queixa urinária e outro por obter frequência inferior ao estipulado.

No estudo de Silva et al. ${ }^{13}$ houve predomínio da IUE entre as mulheres adultas/idosas avaliadas, a maioria multíparas com parto normal e metade delas com história de episiotomia e histerectomia. A IUE também foi predominante nos achados de Henkes et al. ${ }^{14}$, que apontaram uma convivência de $36( \pm 34,33)$ meses com os sintomas da IU antes de terem seu diagnóstico médico estabelecido no serviço especializado. Esta análise não incluiu o tempo de queixa e apenas um dos idosos havia realizado exame diagnóstico específico, o que também reflete o sub-diagnóstico da IU.

Entre os questionários validados, o KHQ mostrou ser o mais utilizado ${ }^{15}$, o que justifica a escolha por ele. Todavia, esta proposta se estendeu a idosos homens, o que pode ter influenciado nos resultados, no entanto, não foram encontrados questionários validados para homens com IU. Apesar da prevalência de queixas urinárias ser inferior em homens quando comparado nas mulheres, Carneiro et al. ${ }^{16}$ reforçam que há alta prevalência em ambos os sexos e que há a necessidade de uma assistência eficaz a cada especificidade. 
Dos domínios do KHQ os com escores mais altos na avaliação inicial, e consequente pior $\mathrm{QV}$, foram impacto da IU $(54,1 \pm 24,8)$, percepção de saúde $(43,7 \pm 11,5)$ e medidas de gravidade $(31,2 \pm 23,8)$, que permaneceram sendo os escores mais altos da avaliação final, porém com valores inferiores, sendo, 29,1 $\pm 11,7,25,0 \pm 13$, 3 e $14,5 \pm 17,0$, respectivamente.

De modo distinto, Oliveira et al. ${ }^{17}$ identificaram escores mais elevados nos domínios percepção geral de saúde, com uma média de 40 pontos, seguido de sono/disposição com 37,47 pontos, impacto da incontinência, com 36,63 pontos e limitações físicas, com 27,48 pontos.

A percepção inicial de saúde variou de 25 a 50 pontos, correspondente a percepção boa a regular, respectivamente (Figura 1). Na avaliação final, apenas 1 idoso manteve percepção regular e também houve um relato de percepção muito boa, havendo repercussão significativa da abordagem proposta $(p=0,001)$. A inexistência de percepções ruins de saúde pode ser resultado do estilo de vida ativa na terceira idade. Já em estudo semelhante ${ }^{17} 40 \%$ das participantes perceberam sua saúde como ruim e $60 \%$ regular, mesmo que as idosas do estudo fizessem parte de um grupo de convivência.
Em relação ao impacto da IU, inicialmente metade dos idosos avaliados pontuou acima de 60 pontos nesse domínio, sendo que um desses pontuou 100, o que significa um grande impacto da IU na vida do idoso. A abordagem prática sobre os MAP possibilitou uma melhora significativa nesse domínio $(\mathrm{p}=0,02)$, de modo que na avaliação final a maioria dos idosos relatou menor interferência da IU.

Uma revisão sistemática realizada por Cestári et al. ${ }^{15}$ aponta diferença nessa interferência conforme o tipo de IU, estando presente na maioria das mulheres com queixa de IUM e IUU. Por outro lado, Fernandes et al. ${ }^{18}$ apontam a IUE por oferecer maior impacto na vida da mulher e constataram que a QV é influenciada, entre outros fatores, pelos anos de perda de urina, IUE e IUU. Isso permite considerar que a IU de modo geral provoca alterações nas várias dimensões da vida de uma mulher, semelhante aos achados desta pesquisa.

Silva et al. ${ }^{13}$ registraram 9 de 11 mulheres que relataram que a IU atrapalha nas atividades diárias. No presente estudo, houve relato de interferência da IU nas atividades de vida diária (AVD) variando de 0 a 33,3 na avaliação inicial e de 0 a 16,6 ao final, demonstrando que houve benefício da proposta fisioterapêutica significativamente $(\mathrm{p}=0,04)$ (Tabela 1$)$.

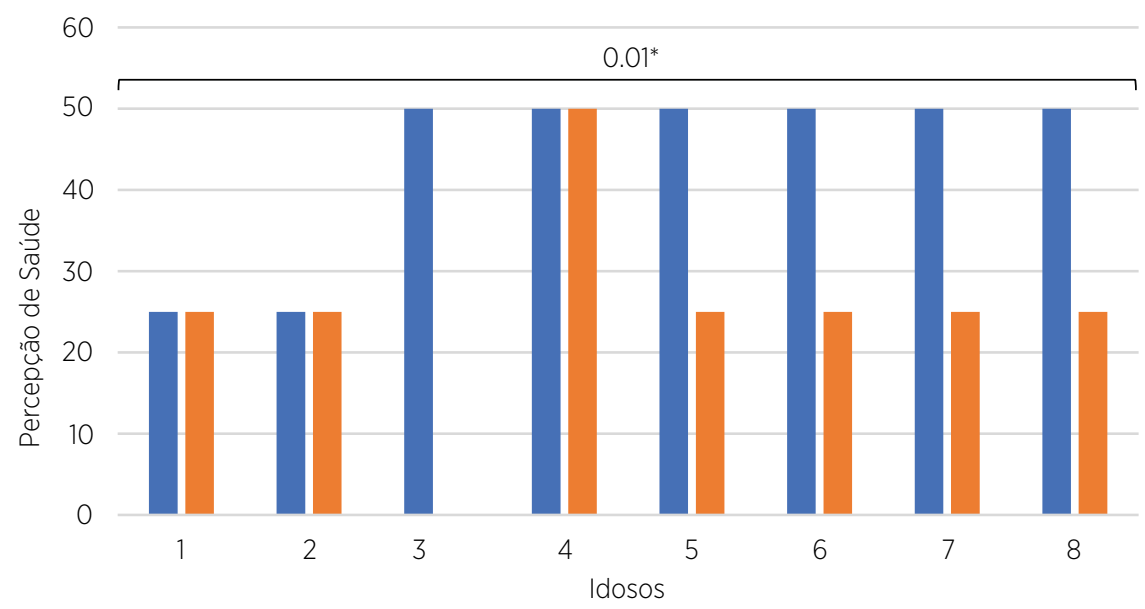

Figura 1. Percepção de saúde dos idosos antes (azul) e após (laranja) participarem de um grupo para abordagem fisioterapêutica da IU em uma UBS em Belém (PA), 2018

Tabela 1. Escores iniciais (I) e finais (F) nos domínios do KHQ apresentados pelos idosos participantes de um grupo para abordagem fisioterapêutica da IU em uma UBS em Belém (PA), 2018

\begin{tabular}{|c|c|c|c|c|c|c|c|c|c|c|c|c|c|c|c|c|c|}
\hline \multicolumn{18}{|c|}{ IDOSOS } \\
\hline \multirow{2}{*}{ Variáveis } & \multicolumn{2}{|c|}{1} & \multicolumn{2}{|c|}{2} & \multicolumn{2}{|c|}{3} & \multicolumn{2}{|c|}{4} & \multicolumn{2}{|c|}{5} & \multicolumn{2}{|c|}{6} & \multicolumn{2}{|c|}{7} & \multicolumn{2}{|c|}{8} & \multirow{2}{*}{ P-valo } \\
\hline & I & $\mathrm{F}$ & I & $F$ & 1 & $F$ & I & $F$ & I & $F$ & I & $F$ & I & $F$ & I & $F$ & \\
\hline LAVD & 33,3 & 16,6 & 0 & 0 & 16,6 & 16,6 & 0 & 0 & 33,3 & 16,6 & 0 & 0 & 33,3 & 0 & 16,6 & 0 & $0,04^{*}$ \\
\hline LF & 16,6 & 0 & 0 & 0 & 33,3 & 0 & 50 & 0 & 0 & 0 & 0 & 0 & 0 & 0 & 16,6 & 0 & 0,06 \\
\hline LS & 11,1 & 0 & 11,1 & 0 & 0 & 0 & 0 & 0 & 22,2 & 0 & 0 & 0 & 0 & 0 & 11,1 & 0 & $0,04^{*}$ \\
\hline $\mathrm{RP}$ & 0 & 0 & MV & MV & MV & MV & MV & MV & 0 & 0 & MV & MV & MV & MV & 0 & $M V$ & 1,0 \\
\hline
\end{tabular}

(continua) 


\begin{tabular}{|c|c|c|c|c|c|c|c|c|c|c|c|c|c|c|c|c|c|}
\hline \multicolumn{18}{|c|}{ IDOSOS } \\
\hline \multirow{2}{*}{ Variáveis } & \multicolumn{2}{|c|}{1} & \multicolumn{2}{|c|}{2} & \multicolumn{2}{|c|}{3} & \multicolumn{2}{|c|}{4} & \multicolumn{2}{|c|}{5} & \multicolumn{2}{|c|}{6} & \multicolumn{2}{|c|}{7} & \multicolumn{2}{|c|}{8} & \multirow{2}{*}{ P-valor } \\
\hline & I & $F$ & I & $F$ & 1 & $\mathbf{F}$ & I & $\mathbf{F}$ & I & $F$ & I & $F$ & 1 & $F$ & I & $F$ & \\
\hline$A E$ & 22,2 & 11,1 & 33,3 & 11,1 & 0 & 0 & 0 & 0 & 11,1 & 11,1 & 0 & 0 & 0 & 0 & 33,3 & 0 & 0,10 \\
\hline SD & 0 & 0 & 50 & 16,6 & 0 & 0 & 0 & 0 & 33,3 & 0 & 33,3 & 16,6 & 0 & 0 & 66,6 & 33,3 & $0,04^{*}$ \\
\hline MG & 8,33 & 0 & 33,3 & 16,6 & 8,3 & 0 & 50 & 0 & 66,6 & 33,3 & 0 & 0 & 50 & 41,6 & 33,3 & 25 & $0,02^{*}$ \\
\hline
\end{tabular}

MV:"missing value"; LAVD: Limitação funcional nas atividades de vida diária; LF: limitação física; LS: limitação social; RP: relações pessoais; AE: aspectos emocionais; SD: sono e disposição; MG: medidas de gravidade.

Quanto à limitação física, muitos dos idosos relataram nenhuma limitação desde o início, resultando em ausência de significância estatística ( $\mathrm{p}=0,06)$, o que pode ter sofrido influência dos exercícios físicos já praticados pelos idosos. Apesar disso, os idosos que relataram alguma limitação baixa ou moderada, zeraram o escore na avaliação final, o que permite entender que o fortalecimento dos MAP atuou como complementar no bem-estar físico dos participantes (Tabela 1). Várias pesquisas com idosos ${ }^{19-21}$ apontaram relação inversa entre prática de atividades físicas e IU, demonstrando relação da queixa com risco à funcionalidade.

Apenas metade dos idosos relatou alguma limitação social, passando de baixa na avaliação inicial a ausente na final, com diferença estatisticamente significativa $(\mathrm{p}=0,04)$ (Tabela 1), o que demonstra a interferência positiva da proposta grupal, dado ratificado por outros autores ${ }^{17}$ que constatam influência moderada da IU sobre a QV e argumentam sobre a importância da participação em atividades em grupo para a socialização.

Nas relações pessoais a avaliação dos idosos apontou escore 0 ou "missing value" (MV), um valor a desconsiderar no processo de cálculo do escore, ou seja, nenhum prejuízo na QV relacionado a esse domínio, e se mantiveram dessa forma até o final dos encontros, não havendo, portanto, diferença significativa $(p=1,0)$, de modo semelhante, Oliveira e Garcia ${ }^{22}$.
Quanto aos aspectos emocionais, houve redução ou manutenção dos escores entre as avaliações, de modo que a abordagem prática não beneficiou significativamente o grupo, apesar de ter sido observado no discurso dos idosos durante a coleta do feedback final. Além disso, similar a outros achados publicados ${ }^{17}$, metade dos idosos referiu nenhuma interferência desde a avaliação inicial, o que também pode ter contribuído para a não significância estatística $(\mathrm{p}=0,1)$.

Metade dos idosos negou interferência no sono e disposição devido a IU, os demais referiram prejuízo leve a moderado, dentre os quais houve melhora parcial ou total $(\mathrm{p}=0,04)$ (Tabela 1$)$.

Apenas um idoso negou fazer uso das medidas de gravidade. Faria et al. ${ }^{23}$ também reportaram a adoção dessas medidas em $90 \%$, demonstrando o quanto a IU afeta o estilo de vida. No presente estudo, houve redução significativa em todos os casos $(p=0,02)$, o que demonstra a grande interferência da fraqueza muscular na adoção dessas medidas, bem como a resposta em curto prazo a abordagem fisioterapêutica (Tabela 1).

Esses benefícios também foram evidentes na redução da frequência urinária, IUE e queixas associadas (dificuldade para urinar) (Tabela 2), demonstrando que o treino proprioceptivo e de força ajudaram no controle miccional.

Tabela 2. Escores iniciais (I) e finais (F) na gravidade dos sintomas, parte 3 do $\mathrm{KHQ}$, apresentados pelos idosos participantes de um grupo para abordagem fisioterapêutica da IU em uma UBS em Belém (PA), 2018

\begin{tabular}{|c|c|c|c|c|c|c|c|c|c|c|c|c|c|c|c|c|c|}
\hline \multicolumn{18}{|c|}{ IDOSOS } \\
\hline \multirow{2}{*}{ Variáveis } & \multicolumn{2}{|c|}{1} & \multicolumn{2}{|c|}{2} & \multicolumn{2}{|c|}{3} & \multicolumn{2}{|c|}{4} & \multicolumn{2}{|c|}{5} & \multicolumn{2}{|c|}{6} & \multicolumn{2}{|c|}{7} & \multicolumn{2}{|c|}{8} & \multirow{2}{*}{ P-valor } \\
\hline & I & $F$ & 1 & $F$ & I & $F$ & I & $F$ & I & $\mathbf{F}$ & I & $F$ & I & $F$ & I & $F$ & \\
\hline \multicolumn{18}{|l|}{ Gravidades dos sintomas } \\
\hline Frequência & 2 & 2 & 1 & 1 & 1 & 0 & 1 & 0 & 3 & 1 & 2 & 1 & 1 & 0 & 1 & 0 & $0,006^{*}$ \\
\hline Noctúria & 3 & 1 & 3 & 1 & 1 & 1 & 0 & 0 & 1 & 1 & 1 & 1 & 1 & 1 & 3 & 1 & 0,07 \\
\hline Urgência & 2 & 1 & 0 & 1 & 3 & 1 & 0 & 0 & 3 & 0 & 1 & 0 & 1 & 0 & 0 & 1 & 0,17 \\
\hline IUU & 1 & 1 & 0 & 0 & 1 & 0 & 0 & 0 & 2 & 0 & 0 & 0 & 1 & 0 & 0 & 0 & 0,10 \\
\hline IUE & 0 & 0 & 0 & 0 & 0 & 0 & 1 & 0 & 3 & 0 & 1 & 0 & 1 & 0 & 3 & 0 & $0,03^{*}$ \\
\hline Enurese noturna & 0 & 0 & 1 & 1 & 0 & 0 & 0 & 0 & 3 & 0 & 0 & 0 & 0 & 0 & 0 & 0 & 0,35 \\
\hline IU no intercurso sexual & 0 & 0 & 0 & 0 & 0 & 0 & 0 & 0 & 1 & 0 & 0 & 0 & 0 & 0 & 0 & 0 & 0,35 \\
\hline
\end{tabular}


Tabela 2. Continuação

\begin{tabular}{|c|c|c|c|c|c|c|c|c|c|c|c|c|c|c|c|c|c|}
\hline \multicolumn{18}{|c|}{ IDOSOS } \\
\hline \multirow{2}{*}{ Variáveis } & \multicolumn{2}{|c|}{1} & \multicolumn{2}{|c|}{2} & \multicolumn{2}{|c|}{3} & \multicolumn{2}{|c|}{4} & \multicolumn{2}{|c|}{5} & \multicolumn{2}{|c|}{6} & \multicolumn{2}{|c|}{7} & \multicolumn{2}{|c|}{8} & \multirow{2}{*}{ P-valor } \\
\hline & I & $\mathbf{F}$ & 1 & $\mathbf{F}$ & I & $\mathbf{F}$ & I & F & I & $\mathbf{F}$ & I & $\mathbf{F}$ & I & $\mathbf{F}$ & 1 & $\mathbf{F}$ & \\
\hline Infecções urinárias frequentes & 0 & 0 & 0 & 0 & 0 & 0 & 0 & 0 & 1 & 0 & 0 & 0 & 0 & 0 & 0 & 0 & 0,35 \\
\hline Dor na bexiga & 0 & 0 & 0 & 0 & 0 & 0 & 0 & 0 & 1 & 0 & 0 & 0 & 0 & 0 & 1 & 0 & 0,17 \\
\hline Outras queixas & 0 & 0 & 1 & 0 & 1 & 0 & 0 & 0 & 1 & 0 & 1 & 0 & 0 & 0 & 1 & 0 & $0,01^{*}$ \\
\hline
\end{tabular}

Uma revisão sistemática realizada por Pereira, Escobar e Driusso ${ }^{24}$ demonstra que, apesar das alterações no envelhecimento que levam a IU terem características estruturais, elas podem ser parcialmente contornadas por meio do tratamento fisioterapêutico, promovendo então a melhora dos sintomas miccionais em mulheres idosas. Assim como em outras propostas semelhantes ${ }^{8,25}$.

Houve significância estatística em parte dos domínios da QV avaliados pelo KHQ dos idosos submetidos à abordagem fisioterapêutica, demonstrando que, mesmo em um nível mais baixo de atenção à saúde é possível ter efeitos sobre a IU e a QV relacionada a esse problema. A maioria dos domínios em que não houve relevância estatística, os valores estiveram baixos na avaliação inicial, o que ampara o argumento de que essa proposta foi ofertada aos idosos em estágio iniciais da IU, auxiliando no controle e prevenção dos agravos relacionados à IU.

Sobre a comparação entre os efeitos do fortalecimento dos MAP aplicados individualmente ou em grupo, um ensaio clínico randomizado ${ }^{26}$ concluiu que a abordagem baseada em grupo não é menos eficaz e apresenta maior relação custo-benefício aos serviços, além disso, Pereira, Escobar e Driusso ${ }^{24}$ apontam que é necessária a continuação dos exercícios, para a manutenção dos benefícios do fortalecimento da musculatura do assoalho pélvico.

Para futuras pesquisas, a fim de aprimorar os resultados, sugerem-se abordagens com maior número ou frequência de encontros em grupo e com avaliação depois de decorrido um tempo maior após as abordagens, como forma de avaliar os efeitos a longo prazo. Também estudos que avaliem quanti e qualitativamente a $\mathrm{QV}$.

Em relação aos serviços de saúde, recomenda-se esse tipo de proposta na rotina do serviço, de modo que a educação em saúde seja sempre ofertada periodicamente a usuários e profissionais, e que a figura do fisioterapeuta esteja sempre presente e a frente de práticas preventivas como esta, visando minimizar a ocorrência de casos graves de IU e a negligência frente a esta queixa tão relevante à funcionalidade do idoso.

\section{CONSIDERAÇÕES FINAIS}

Este estudo apontou os benefícios de uma abordagem educativa e prática voltada para o fortalecimento dos MAP em idosos com queixas urinárias relacionadas à IU, sendo mais evidente na melhora da percepção de saúde e na redução do impacto da IU na vida do idoso, limitação nas AVD e na vida social, interferência no sono e disposição, nas medidas de gravidade, frequência urinária e ocorrência de queixas associadas e IUE, isto é, em vários domínios da $\mathrm{QV}$ dos idosos, mostrando-se efetiva no manejo da IU em estágios iniciais e na prevenção da IU, tão recorrente na população idosa.

\section{REFERÊNCIAS}

1. Kessler M, Facchini LA, Soares MU, Nunes BP, França SM, Thumé E. Prevalência de incontinência urinária em idosos e relação com indicadores de saúde física e mental. Rev Bras Geriatr Gerontol. 2018;21(4):409-19. doi: 10.1590/1981-22562018021.180015.

2. Secretaria de Estado da Saúde do Paraná. Linha guia da saúde do idoso. Curitiba: Sesa; 2017.

3. Ribeiro CR, Tavares DMS, Ferreira PCS, Dias FA, Ferreira LA. Fatores asssociados à incontinência urinária entre idosos da zona rural. Rev Enferm Atenção Saúde. 2018;7(1):3-14. doi: 10.18554/reas.v7i1.1832.

4. Marques LP, Schneider IJC, Giehl MWC, Ledur Antes D, D’orsi E. Fatores demográficos, condições de saúde e hábitos de vida associados à incontinência urinária em idosos de Florianópolis, Santa Catarina. Rev Bras Epidemiol. 2015;18(3):595-606. doi: 10.1590/1980-5497201500030006.

5. Associação Portuguesa de Urologia. Dossier Incontinência Urinária [Internet]. Lisboa: Monstros e Companhia; 2014 [cited 2018 Jun 22]. Available from: http://www.apurologia.pt/incontinencia/ incontinencia_2014/Dossier_Inc_Urinaria_2014.pdf

6. Silva JCP, Soler ZASG, Wysocki AD. Fatores associados à incontinência urinária em mulheres submetidas ao exame urodinâmico. Rev Esc Enferm USP. 2017;51:e03209. doi: 10.1590/s1980-220×2016140903209.

7. Tomasi AVR, Santos SMA, Honório GJS, Locks MOH. Incontinência urinária em idosas: práticas assistenciais e proposta de cuidado âmbito da atenção primária de saúde. Texto \& Contexto Enferm. 2017; 26(2). doi: 10.1590/0104-07072017006800015. 
8. Silva RMN. Efetividade da cinesioterapia aplicada na incontinência urinária feminina - revisão integrativa [monografia]. Juiz de Fora: Universidade Federal de Juiz de Fora; 2017.

9. Figueiredo EM, Baracho SM, Vaz CT, Sampaio RF. Educação de funcionárias de unidade básica de saúde acerca da atenção fisioterapêutica na incontinência urinária: relato de experiência. Fisioter Pesqui. 2012;19(2):103-8. doi: 10.1590/S1809-29502012000200003.

10. Fonseca ESM, Camargo ALM, Castro RA, Sartori MGF, Fonseca MCM, Lima GR, et al. Validação do questionário de qualidade de vida (King's Health Questionnaire) em mulheres brasileiras com incontinência urinária. Rev Bras Ginecol Obstet. 2005;27(5):235-42

11. Pinceli MG, Moccellin AS. Protocolos de prevenção da incontinência urinária em idosas: revisão crítica da literatura. Geriatr Gerontol Aging. 2014;8(2):131-5. Available from: http://ggaging.com/ details/103/pt-BR

12. Abrams P, Cardozo L, Khoury S, Wein A. (ed). Incontinence. International Consultation on Urological Diseases. European Association of Urology. 5th ed. Bristol: Icud; 2013.

13. Silva LWS, Lucas TQC, Santos SSO, Novaes VS, Pires EPOR, Lodovici FMM. Fisioterapia na incontinência urinária: olhares sobre a qualidade de vida de mulheres idosas. Rev Kairós. 2017;20(1):221-38. doi: 10.23925/2176-901X.2017v20i1p221-238.

14. Henkes DF, Fiori A, Carvalho JAM, Tavares KO, Frare JC. Incontinência urinária: o impacto na vida de mulheres acometidas e o significado do tratamento fisioterapêutico. Semina Cienc Biol Saude. 2015;36(2):45-56.

15. Cestári CE, Souza THC, Silva AS da. Impacto da incontinência urinária na qualidade de vida de idosas. Rev Ciênc Estud Acad Med. 2017;7:27-37.

16. Carneiro JA, Ramos GCF, Barbosa ATF, Medeiros SM, Lima CA, Costa FM, Caldeira AP. Prevalência e fatores associados à incontinência urinária em idosos não institucionalizados. Cad Saúde Colet. 2017;25(3):268-77. doi:10.1590/1414-462x201700030295.

17. Oliveira GSM, Botaro NAAB, Botaro CA, Rocha CQ. Análise da incontinência urinária na qualidade de vida de idosas frequentadoras de um grupo de convivência social em Muriaé-MG. Rev Pesq Fisioter. 2014;4(1):7-15. doi: 10.17267/2238-2704rpf.v4i1.379.

18. Fernandes S, Coutinho EC, Duarte JC, Nelas PAB, Chaves CMCB, Amaral O. Qualidade de vida em mulheres com Incontinência Urinária. Rev Enferm. 2015;4(5):93-9. doi: 10.12707/RIV14042.

19. Lee AH, Hirayama F. Physical activity and urinary incontinence in older adults: a community-based study. Current Aging Sci. 2012;5(1):35-40. doi: 10.2174/1874612811205010035.

20. Menezes EC, Virtuoso JF, Mazo GZ. Older women with urinary incontinence present less physical activity level usual. Rev Bras Cineantropom Desempenho Hum. 2015 ;17(5):612-20. doi: 1980-0037.2015v17n5p612.

21. Parker-Autry C, Houston DK, Rushing J, Richter HE, Subak L, Kanaya AM, Kritchevsky SB. Characterizing the functional decline of older women with incidente urinary incontinence. Obstet Gynecol. 2017;130:1025-32. doi: 10.1097/ AOG.0000000000002322.

22. Oliveira JR, Garcia RR. Cinesioterapia no tratamento da Incontinência Urinária em mulheres idosas. Rev Bras Geriatr Gerontol. 2011;14(2):343-51. doi: 10.1590/S1809-98232011000200014.

23. Faria CA, Menezes AMN, Rodrigues AO, Ferreira ALV, Bolsas CN. Incontinência urinária e noctúria: prevalência e impacto sobre a qualidade de vida em idosas numa Unidade Básica de Saúde. Rev Bras Geriatr Gerontol. 2014;17(1):17-25. doi: 10.1590/S1809-98232014000100003.

24. Pereira VS, Escobar AC, Driusso P. Efeitos do tratamento fisioterapêutico em mulheres idosas com incontinência urinária: uma revisão sistemática. Rev Bras Fisioter. 2012;16(6):463-8. doi: 10.1590/S1413-35552012005000050.

25. Jahromi MK, Talebizadeh M, Mirzaei M. The effect of pelvic muscle exercises on urinary incontinency andm self-Esteem of elderly females with stress urinary incontinency, 2013. Global J Health Sci. 2015; 7(2):71-9. doi: 10.5539/gjhs.v7n2p71.

26. Dumoulin C, Morin M, Mayrand MH, Tousignant $M$, Abrahamowicz M. Group physiotherapy compared to individual physiotherapy to treat urinary incontinence in aging women: study protocol for a randomized controlled trial. Trials. 2017;18:544. doi: 10.1186/s13063-017-2261-4. 